G.C. Gebel, T. Mosbach, W. Meier, M. Aigner, Laser-Induced Blast Waves in Air and their Effect on Monodisperse Droplet Chains of Ethanol and Kerosene, Shock Waves 25 (2015) 415-429.

The original publication is available at www.springerlink.com

http://dx.doi.org/10.1007/s00193-015-0564-5 


\title{
Laser-Induced Blast Waves in Air and their Effect on Monodisperse Droplet Chains of Ethanol and Kerosene
}

\author{
G. C. Gebel \\ T. Mosbach \\ W. Meier \\ M. Aigner
}

Institute of Combustion Technology, German Aerospace Center (DLR), Pfaffenwaldring 38-40, 70569 Stuttgart, Germany

G. C. Gebel

E-mail: Gregor.Gebel@dlr.de

\begin{abstract}
Weak spherical blast waves in static air and their breakup of ethanol and Jet A-1 kerosene droplets were investigated. The blast waves were created by laser-induced air breakdowns at ambient temperature and pressure. In the first step of this study they were visualized with schlieren imaging, and their trajectories were tracked with high temporal resolution. The laser pulse energy was varied to create blast waves of different strengths. Their initial energies were determined by the application of a numerical and a semi-empirical blast wave model. In the second step monodisperse ethanol and kerosene droplet chains were injected. Their interaction with the blast waves was visualized by the application of shadowgraph imaging. The perpendicular distance of the breakdown origin towards the droplet chains was varied to study the effect on the fuel droplets as a function of the distance. Droplets within a few millimeters around the breakdown origin were disintegrated into two to three secondary droplets. The blast-induced flow velocities on the post-shock side and the corresponding Weber numbers were calculated from the data of a non-dimensional numerical simulation, and a close look was taken at the breakup process of the droplets. The analysis showed that the aerodynamic force of the blast-induced flow was sufficient to deform the droplets into disk-like shapes, but diminished too fast to accomplish aerodynamic breakup. Due to the release of strain energy, the deformed droplets relaxed, stretched into filaments and finally disintegrated by capillary pinching.
\end{abstract}

\section{Keywords}

Blast wave, Laser-induced breakdown, Droplet breakup, Flow visualization, Schlieren, Shadowgraph

\section{Introduction}

Advanced combustion systems are needed for modern aviation gas turbines to achieve high efficiency and low emissions. Their development can benefit significantly from tailor-made numerical tools. 
Compared with iterative prototype testing, numerical simulations allow parameter surveying and geometry optimization with faster turnaround times and at much lower cost. For fuel spray ignition, however, numerical tools have not yet matured. Spray ignition is a complex process involving multiple mechanisms related to the research fields of fluid dynamics, two-phase flows, heat transfer, evaporation, chemical kinetics, and plasma physics. With the limitations of present numerical tools, the transition from an ignition spark into a flame kernel is only computable if the real physics are strongly simplified. Therefore, a better understanding of the involved mechanisms is necessary to develop more accurate models. Experiments in laboratory setups with well-defined boundary conditions can provide very important contributions. Combustion engines typically ignite by electrical spark discharge. But spark plugs are inconvenient in laboratory setups for the following reasons. Shifting of the breakdown position is restricted. Boundary conditions are complicated, because the plug acts as a heat sink and interacts with the flow field. Variation of the breakdown energy is restricted, and the triggering accuracy is low. Ignition by non-resonant laser-induced breakdown is a good alternative, as it is advantageous in all the stated aspects above. Both ignition concepts feature similar physical properties, particularly the electron cascade mechanism [1], and involve the formation of a blast wave, as demonstrated in Figure 1. It shows schlieren images from a laserinduced breakdown, an inductive and a capacitive spark discharge in air. The density gradients at the blast wave shock fronts are clearly visible. In our previous publications we investigated the features of laser-induced breakdowns and the ignition of liquid fuels [2-4]. We found that spherical blast waves formed by the rapid initial expansion of the breakdown plasmas play an important role in the ignition process, because they induce secondary droplet breakup [3].

Indeed, the secondary breakup of droplets in high velocity flow fields has been extensively investigated over almost seven decades, and reviews are given in the papers by Pilch and Erdman [5], Gelfand [6] and Guildenbrecher et al. [7]. Experiments were conducted in different types of test facilities, including shock tubes, drop towers and blowdown wind tunnels. The paper by Guildenbrecher et al. includes a review on the specific characteristics of the different types of test facilities. It is necessary to emphasize that the flow conditions in different experiments varied significantly, and consequently different droplet behaviors and breakup mechanisms were identified. The most disruptive breakup mechanisms were observed at supersonic flow conditions, see for instance the publications [8-12], where droplets were exposed to steady blast-induced flows for up to several milliseconds. Although we also investigate droplet breakup in a blast-induced flow in this study, the basic conditions are different. In the Eulerian frame of reference, the blast-induced flow on the post-shock side of a spherical blast wave is not steady, but decays rapidly and eventually turns into a weak rarefaction wave. In the Lagrangian frame of reference, the expansion velocity of the shock front converges towards sonic velocity, the wave turns into an acoustic wave. Summarizing, droplets exposed to spherical blast waves face highly transient flow conditions, and apart from our previous paper [3], we are not aware of any other study regarding the effect of spherical blast waves on droplets. Our previous paper provided microscopic images of kerosene droplets in the vicinity of a laser-induced breakdown. The breakup by the blast-induced flow was observed $10 \mathrm{~mm}$ below the 
breakdown origin with a very high spatial and temporal resolution. The investigation presented in this paper continued our previous work. The focus was on the effect of laser-induced blast waves on fuel droplets at different radial distances from the breakdown origin. The investigation was divided into three parts, which are reflected by the structure of the paper. In the first part, the trajectories of spherical blast waves were tracked with high temporal resolution by schlieren image sequences. Four different methods to determine the energies from the trajectories were tested. Two of them were selected and validated on blast waves of different strengths. The investigation provided the initial blast wave energy $E_{0}$, which was required to calculate the droplet Weber numbers in the blast-induced flow on the post-shock side. In the second part, the blast wave interaction with monodisperse droplet chains of ethanol and Jet A-1 kerosene was investigated with shadowgraph imaging. The blast wave strength was kept constant at a high level. Different geometrical configurations were investigated, including single droplet chains and five chains in a row at different horizontal distances from the breakdown origin. Finally, calculations of the blast-induced flow velocities and Weber numbers were performed on the basis of the determined blast wave energy. This allowed a comparison of the observed droplet breakup with conventional breakup models. Enlarged views of droplets at different horizontal distances from the breakdown origin gave insight into the mechanism behind the observed breakup.
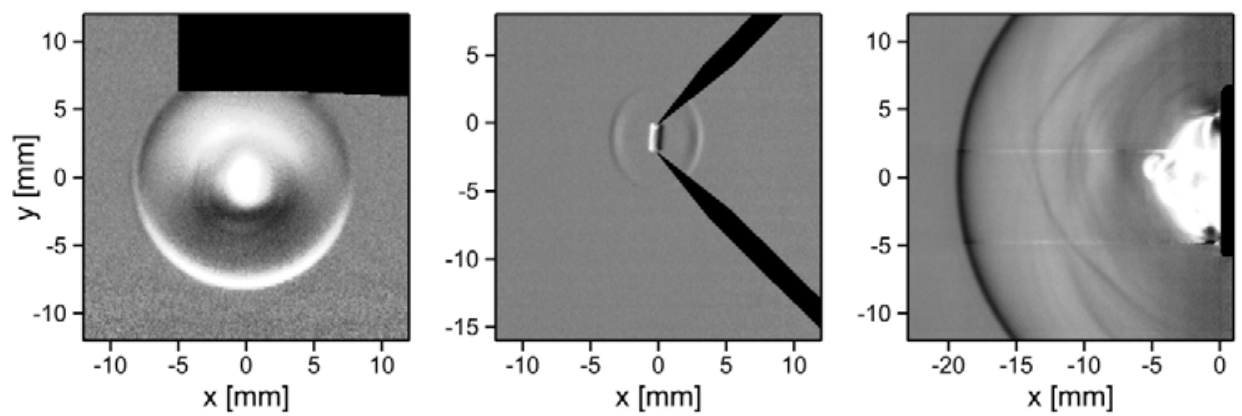

Fig. 1 Exemplary Schlieren images, taken several microseconds after a laser-induced breakdown (left), an inductive (center) and a capacitive spark discharge (right) in ambient air. The blast waves are clearly visible.

\section{Experimental setup}

\subsection{Test rig and injector}

Experiments were performed in a vertically arranged flow channel with a square cross section of $62 \mathrm{~cm}^{2}$ and a length of $1 \mathrm{~m}$. A schematic and a cutaway are shown in Figure 2. Three sides were equipped with windows with anti-reflective coating. The fourth side was attached to a two-axis 
traverse system for horizontal and vertical positioning. A downwards directed droplet chain injector model TSI 3450 was installed at approximately one fourth height of the flow channel. Droplets were generated by the induced Plateau-Rayleigh capillarity instability mechanism [13]: A piezoelectric actuator induced a sustained regular distortion on the fuel jets which streamed out of the orifice holes. Two orifices were available, one with a centered $50 \mu \mathrm{m}$ hole, and one with five coplanar $50 \mu \mathrm{m}$ holes spaced at a centre-to-centre distance of $1 \mathrm{~mm}$. According to Rayleigh [13], the excitation wavelength to obtain a monodisperse droplet breakup is given by:

$$
\lambda=4.508 D_{j}
$$

In Equation (1), $\lambda$ is the excitation wavelength and $D_{j}$ is the diameter of an undisturbed fuel jet. For incompressible fuels the jet diameter can be replaced by the hole diameter $D_{h}$. The excitation frequency is then given by

$$
v=\frac{u_{j e t}}{4.508 D_{h}}
$$

with the fuel jet velocity

$$
u_{j e t}=\frac{4 \dot{m}_{f}}{\rho_{f} \pi \times D_{h}^{2}} .
$$

In Equations (2) and (3), $\dot{m}_{f}$ and $\rho_{f}$ are the total fuel mass flow for all holes and the fuel density, respectively. The number of holes is given by $x$. The droplet diameter $D_{d}$ and the center-to-center distance between two successive droplets $d_{d}$ can be calculated by geometrical means:

$$
\begin{aligned}
& D_{d}=\left(\frac{6 \dot{m}_{f}}{\rho_{f} \pi x v}\right)^{\frac{1}{3}}, \\
& d_{d}=\frac{2 D_{d}^{3}}{3 D_{h}^{2}} .
\end{aligned}
$$

The fuel was supplied by a siphon bottle with an inner and a circumferential chamber. The inner chamber contained the fuel, the circumferential chamber was perfused by a water flow with a controlled temperature. Thus, the fuel temperature could be controlled with an accuracy of $\pm 0.2 \mathrm{~K}$. 
Inflowing air displaced the fuel and drove it to the injector. The flow channel could be vented by a steady top down air flow, in order to flush out fuel vapour to prevent unwanted ignition and uncontrolled combustion. The air flow and fuel vapour were exhausted sideways at the bottom of the flow channel. Liquid fuel was captured with a funnel and collected in a steel bottle at the very bottom.
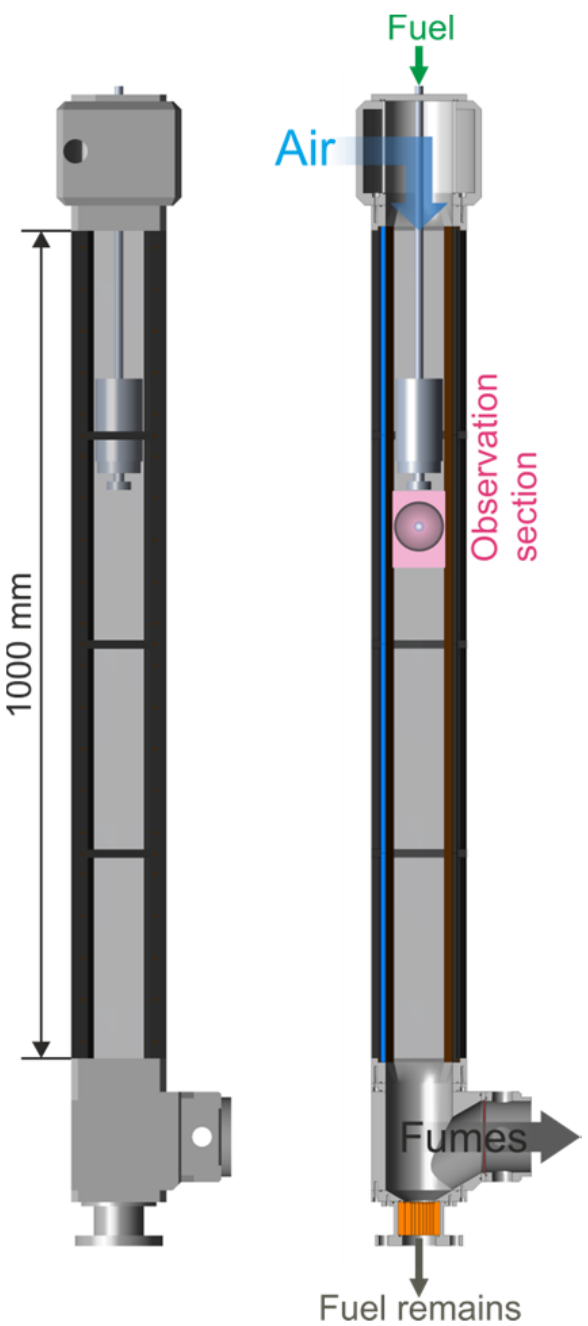

Fig. 2 Schematic of the flow channel, outside view (left) and cutaway (right).

\subsection{Laser-induced air breakdowns}

Breakdowns were created by a flash lamp-pumped, Q-switched and frequency-doubled Nd:YAG laser (model InnoLas SpitLight 600-10). It provided Gaussian (spatial and temporal) pulses of up to $300 \mathrm{~mJ}$ and a trigger accuracy of $\pm 1 \mathrm{~ns}$ in the single pulse mode. The pulse energy was adjusted by means of the delay between the flash lamp and the Q-switch. The beam path is illustrated in Figure 3. 
Laser pulses were steered to an optical bench with a periscope and expanded to a diameter of approximately $40 \mathrm{~mm}$ with a Galilean telescope. Afterwards, they were directed into the flow channel with a downward angle of 23 degrees and refocused directly in front of the inlet window by a lasergrade doublet lens with a focal length of $120 \mathrm{~mm}$. Non-resonant multiphoton ionization at the focal point generated electron cascade breakdowns with high ionization levels. This process is described in more detail by Ronney [1]. The initial supersonic expansion of the breakdown plasma caused the formation of a spherical blast wave, which detached from the breakdown after approximately 1 to $2 \mu \mathrm{s}$.

\subsection{Schlieren and shadowgraph setup}

A schematic of the experimental setup is given in Figure 3. The light beam from a halogen projector lamp was collimated with a plano-convex lens $(f=300 \mathrm{~mm})$ to a diameter of approximately $90 \mathrm{~mm}$. It passed through the observation section inside the flow channel and was refocused by another plano-convex lens $(f=600 \mathrm{~mm})$ on the opposite side. A monochrome Photron / LaVision High Speed Star 6 high-speed CMOS camera recorded schlieren images at a sustained repetition rate of $25 \mathrm{kHz}$, an exposure time of $1 \mu \mathrm{s}$ and a resolution of $448 \times 448$ pixels. The camera gate function is shown in Figure 4. It features a fast rising edge, a slower falling edge and an overall time span of $1.09 \mu \mathrm{s}$. The temporal jitter is approximately $\pm 40 \mathrm{~ns}$. The $50 \%$ effective exposure time is the time when half of the integral over the gate function has passed, which is $510 \mathrm{~ns}$. This information is particularly important for the blast wave energy determination in section 3.1.

Two different camera lenses were used: A Tokina $100 \mathrm{~mm} \mathrm{f} / 2.8$ lens (for schlieren images of blast waves) and a Nikkor $180 \mathrm{~mm} \mathrm{f} / 2.8$ lens (for shadowgraph images of droplet chains). The camera and the laser were externally triggered by a combination of a BNC 565 and a BNC 555 pulse generator. The BNC 565 gave the continuous trigger signals for the camera controller and for the $10 \mathrm{~Hz}$ laser flash lamp. The BNC 555 was synchronized with the BNC 565 and gave the singular trigger signals for the laser Q-switch and the camera start. The repetition rate of $25 \mathrm{kHz}$ was not sufficient to capture the very fast fluid mechanical processes in a single recording. Therefore, multiple recordings were performed, and the delay between the Q-switch trigger and the camera start was sequentially increased.

The Schlieren effect was generated with an iris aperture, which was adjusted to cut with one lamella into the focal point from one side, in order to visualize vertical density gradients. An OG570 longpass filter in front of the aperture suppressed chromatic aberration. The majority of the breakdown radiation was blocked by the aperture, but the remaining radiation could still saturate the camera's CMOS sensor. Therefore, broadband transmission filters were mounted in front of the camera lens for the recordings between 2 and $8 \mu$ s after the laser pulse. The transmission of the filters ranged from $\tau=0.125$ to 0.5 .

For shadowgraph imaging, the aperture and the filters were removed from the light beam path. The camera was shifted closer to the flow channel to obtain a greater magnification. In contrast to the 
schlieren setup, the shadowgraph setup visualized fuel droplets with great sharpness, while blast waves were not clearly visible. The radiation from the breakdown plasma was blocked with a small screen to protect the CMOS sensor from damage.

The pressure, temperature and humidity of the ambient air were regularly measured during the experiment. Variations were very small, the temperature ranged between 22.6 and $23.8{ }^{\circ} \mathrm{C}$, the pressure between 978.1 and $978.7 \mathrm{hPa}$, and the humidity between 27.5 and $28.4 \%$. Consequently, the calculated sonic velocity ranged between 345.7 and $346.4 \mathrm{~m} / \mathrm{s}$. The data were applied in the calculations of the blast wave energies and blast-induced flow velocities.

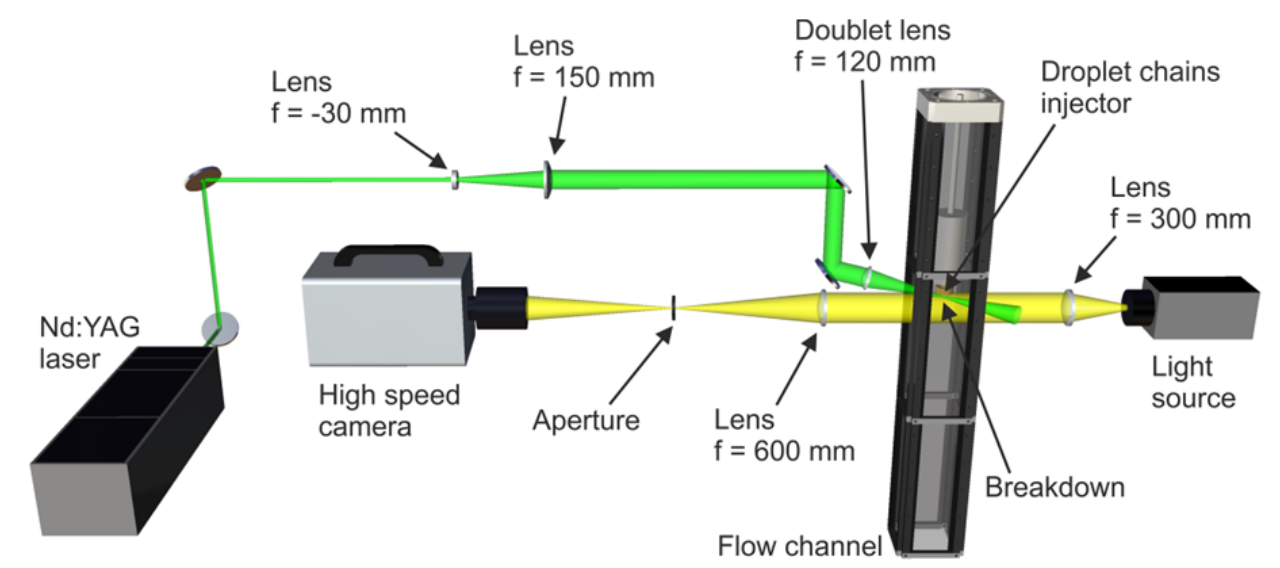

Fig. 3 Experimental setup with laser beam path (green) and light beam path for schlieren and shadowgraph imaging (yellow).

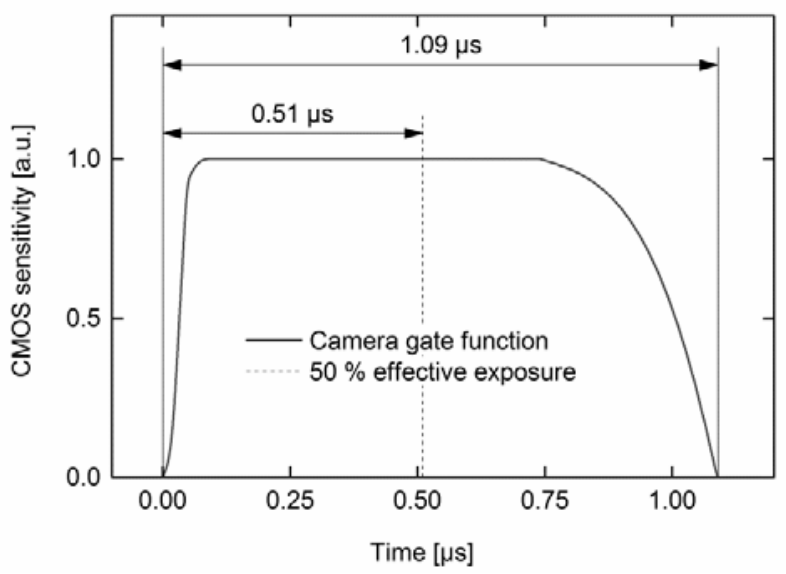

Fig. 4 Camera gate for a configured exposure time of $1 \mu \mathrm{s}$. 


\section{Measurements, post-processing, and analysis}

\subsection{Laser-induced blast waves in air}

Laser-induced blast waves in ambient air were investigated with the schlieren imaging technique. Three recordings were taken for every selected laser pulse energy and delay, in order to account for statistical scattering. The intention of this investigation was to identify a method to determine accurate blast wave energies from the expansion trajectories. Blast waves of five different strengths were created by varying the laser pulse energy. The pulse energy was adjusted by means of the delay between the flash lamp and the Q-switch trigger: A delay of $215 \mu$ s provided the maximum laser pulse energy, whereas a delay of $335 \mu$ s provided just enough energy to create laser-induced breakdowns at the focal point. The adjusted delays were $215,255,295,315$, and $335 \mu$ s. Only a fraction of the laser pulse energy was absorbed in the breakdown. During the first few nanoseconds of the pulse, many of the photons passed the breakdown region, because the plasma was partially transparent and did not fully occupy the focal point, see Chen et al. for more details [14]. Therefore, the breakdown energy was determined in a two-step measurement with a volume absorber type Gentec-EO UP19K-15S-VRD0. Average energies over 2000 pulses were separately measured $20 \mathrm{~mm}$ in front of and behind the focal point. Energy losses due to scattering and diffraction are negligible in laser-induced breakdowns [15]. Therefore, the difference between the pulse energy $E_{p}$ and its transmitted fraction behind the breakdown $E_{t}$ virtually equaled the breakdown energy $E_{b}$. Table 1 gives the average pulse and breakdown energies for five different Q-switch delays $\Delta t$, along with the FWHM (full width at half maximum) pulse lengths and the standard deviations $s_{b}$ of the breakdown energies. Minor FWHM fluctuations between individual pulses occurred for Q-switch delays of 315 and $335 \mu$ s, which are indicated by the given accuracy of $\pm 1 \mu \mathrm{s}$. The small standard deviations indicate that the total pulse energies were not significantly affected by the fluctuations.

Representative schlieren images for five different breakdown energies 10, 24, and $48 \mu$ s after the laser pulse are shown in Figure 4. Spherical blast waves are clearly visible as circular density gradients. The white spot at the origin is the breakdown plasma, which is visible for several microseconds. The breakdown is surrounded by a plume of compressed, hot gas. After the breakdown has decayed, the gas plume expands slowly and mixes with the surrounding air. Blast wave trajectories were determined from the schlieren images with a MATLAB algorithm. It created synthetic blast wave images with various radii and cross-correlated them with the schlieren images. With regard to the high expansion velocities of the blast waves, the camera exposure time of $1 \mu \mathrm{s}$ was relatively long. Consequently, the shock fronts appeared somewhat blurred in the schlieren images. This challenge was overcome by the interpolation of the radius at the $50 \%$ effective exposure time from the cross-correlation peak distribution. Consequently, the blast wave radii were determined with a theoretical accuracy of $\pm 0.05 \mathrm{~mm}$, which was about $\pm 1 / 2$ pixel of the image resolution. The resulting 
trajectories are shown in Figure 5. They are averages over three datasets. Error bars are not displayed, because the reproducibility was very good. The low curvature of the trajectories indicates that the blast waves were observed during their transitions from strong blasts into acoustic waves.

Literature provides several expansion models which allow the determination of the initial energy $E_{0}$ from the trajectory. Four models were applied to the blast wave trajectory of the $245.6 \mathrm{~mJ}$ breakdown. A widely used model is the Taylor-Sedov solution, which is given as [16]:

$$
r(t)=\left[\frac{E_{0} t^{2}}{K(\gamma) \rho_{0}}\right]^{\frac{1}{5}}
$$

In Equation (6), $r$ is the radius, $t$ the time, and $\rho_{0}$ the density of the unshocked air. The constant $K$ is 0.856 for $\gamma=1.4$ [17].

In the experiment, blast waves were investigated as they expanded into ambient air at atmospheric conditions. The only effect that could have heated the air in the vicinity of the breakdown and therefore affected $\gamma$ is the absorption of plasma radiation emitted by the breakdown. This effect is negligible at the investigated distances from the breakdown surface, as demonstrated in our recently published numerical simulation of a laser-induced air breakdown, see Joarder et al. [4]. Consequently, $\gamma=1.4$ was applied in all analyses in the course of this study.

Equation (6) is a self-similar solution to the blast wave problem. It assumes a very high pressure gradient over the shock front, so that the pressure of the unshocked air $p_{0}$ is set to zero in the Rankine-Hugoniot relations [18]. This assumption is sufficient only for a short time. A spherical blast wave decelerates with its increasing radius, and the pressure and temperature gradients across the shock front decrease. This is caused by the geometrical growth of the spherical shock front and the associated energy density decrease, and by dissipation. The latter is particularly relevant at the early stage, when the pressure gradient is still high. Consequently, $p_{0}$ becomes relevant within several nanoseconds, and Equation (6) predicts an unnatural decrease of the shock front velocity into the subsonic regime. This is demonstrated by the blue graph in Figure 6: The Taylor-Sedov solution was fitted to the measured trajectory with an in-house LabVIEW algorithm, applying the method of least $\Delta y$-squares. The agreement was of poor quality, and the Taylor-Sedov solution was obviously not applicable for the present measurements. Actually it is only accurate for strong blasts, such as nuclear detonations [17]. The laser-induced blast waves created in our experiments were only strong for several nanoseconds, and the measured trajectories covered the transition from strong blasts into acoustic waves. This is well illustrated in Figure 6 by the fact that the trajectory is nearly parallel to the line for a Mach number of $M a=1$. Therefore, three self-similar models were applied which were developed for the transition regime: The models by Sedov [18] and Brode [19] are numerically derived, non-dimensional trajectories. The model by Jones [20] is a set of semi-empirical equations 
for planar shocks and cylindrical and spherical blast waves. Jones' equations for spherical blast waves are:

$$
\begin{aligned}
& \tau=0.543\left[\left(1+4.61 x(t)^{\frac{5}{2}}\right)^{\frac{2}{5}}-1\right], \\
& \tau=c_{0} \frac{t}{r_{0}} \\
& x(t)=\frac{r(t)}{r_{0}} \\
& r_{0}=\left[\frac{6.25 E_{0}}{B \gamma p_{0}}\right]^{\frac{1}{3}} .
\end{aligned}
$$

Here, $\tau$ is the non-dimensional time, $t$ is the dimensional time, $r$ is the radius, $r_{0}$ is a reference radius, $C_{0}$ is the sonic velocity, and $\gamma$ is the isentropic exponent. $B$ is a geometry parameter, values for different cases are given in [21]. The models were iteratively fitted to the measured trajectory for a breakdown energy of $245.6 \mathrm{~mJ}$ with an in-house LabVIEW algorithm. The increment of the iterations was $0.01 \mathrm{~mJ}$, the best fits are displayed in Figure 6. The qualities of the fits are indicated by the sums of the least $\Delta y$-squares. Sedov's model gave a blast wave energy of $151.0 \mathrm{~mJ}$ and a relatively high $\Sigma \Delta y_{i, \text { Sedov }}{ }^{2}$ of $19.71 \mathrm{~mm}^{2}$. In contrast, Jones' model gave a $\Sigma \Delta y_{i, \text { Jones }}{ }^{2}$ of $7.73 \mathrm{~mm}^{2}$ and Brode's model gave a $\Sigma \Delta y_{i, \text { Brode }}{ }^{2}$ of only $4.1 \mathrm{~mm}^{2}$. Consequently, only the models of Jones and Brode were applied to the trajectories of the four weaker blast waves.

The blast wave energy determination from the expansion trajectory was highly sensitive to measurement inaccuracies, and therefore a careful error analysis was performed. Possible error sources were the time assignments and the calibrated scales of the schlieren images. The temporal accuracy was determined to be better than $\pm 40 \mathrm{~ns}$ and the scale accuracy to be better than $\pm 0.1 \%$. The results of the energy determination and error analysis are given in Table 2 and displayed in Figure 7. Table 2 gives the blast wave energies together with their maximum deviations, the blast wave energy proportions in relation to the breakdown energy and the sums of the least $\Delta y$-squares. Error bars in Figure 7 indicate the maximum deviations.

Concluding, the blast waves consume a significant amount of the breakdown energies, which is approximately $52 \%$ at a breakdown energy of $245.6 \mathrm{~mJ}$ and steadily increases to $77 \%$ at a breakdown energy of $11.6 \mathrm{~mJ}$. The models of Brode and Jones feature a very good agreement for all 
investigated breakdown energies and do not disagree by more than $4.2 \%$ (determined for $\left.E_{b}=89.6 \mathrm{~mJ}\right)$. Therefore, we regarded the two models as successfully validated within our experimental possibilities, and assumed that a blast wave energy of $52 \%$ for a laser pulse energy at $\sim 246 \mathrm{~mJ}$ was sufficiently close to reality to use it in our subsequent analysis, see section 3.3.

Our results are in good agreement with the study by Brieschenk et al. [22], who investigated the blast wave expansion from laser-induced breakdowns created by a Q-switched ruby laser and a focusing lens with a focal length of $100 \mathrm{~mm}$. The laser pulse energy ranged between 100 and $2700 \mathrm{~mJ}$. Blast wave energy fractions were determined by the numerical integration of the RankineHugoniot relations using Dewey's empirical trajectory model [23]. They were found to be 50 to $65 \%$ of the laser pulse energies. Moreover, our results are in very good agreement with the study by Phuoc and White [24]. They investigated the blast wave expansion from laser-induced breakdowns created by a Q-switched Nd:YAG-laser at $1064 \mathrm{~nm}$ and a focusing lens with a focal length of $120 \mathrm{~mm}$. They determined blast wave energies during the first few microseconds after the laser pulse using the Taylor-Sedov solution. Breakdown energies ranged between 15 and $50 \mathrm{~mJ}$. At $50 \mathrm{~mJ}$, the blast wave energy was $57 \%$ of the breakdown energy. Its proportion increased with decreasing breakdown energy, and $70 \%$ were determined at $15 \mathrm{~mJ}$. The trend and the order of magnitude are very similar to our results. In particular, they determined a blast wave energy ratio of $61.6 \%$ for a breakdown energy of $44 \mathrm{~mJ}$, while we determined $62.6 \%$ (Brode's model) and $64.9 \%$ (Jones' model) for a breakdown energy of $46.1 \mathrm{~mJ}$. Our experiment and analysis presented in this section were required for the analysis of droplet breakups in section 3.3, because Phuoc and White applied maximum breakdown energies of $50 \mathrm{~mJ}$, while we investigated droplet breakups at breakdown energies of almost $250 \mathrm{~mJ}$.

Tab. 1 Average laser pulse energies, estimated breakdown energies, breakdown energy standard deviations over 2000 laser pulses and laser pulse FWHM length.

\begin{tabular}{lccccc}
\hline Laser Q-switch $\Delta t[\mu \mathrm{s}]$ & 335 & 315 & 295 & 255 & 215 \\
\hline Laser pulse FWHM $[\mathrm{ns}]$ & $14.3 \pm 1$ & $10 \pm 1$ & 8.2 & 6.5 & 6 \\
Laser pulse $E_{p}[\mathrm{~mJ}]$ & 24.2 & 60.7 & 104.9 & 199.6 & 276.6 \\
Air breakdown $E_{b}[\mathrm{~mJ}]$ & 11.6 & 46.1 & 89.6 & 176.1 & 245.6 \\
Air breakdown $s_{b}[\mathrm{~mJ}]$ & 0.75 & 0.56 & 0.75 & 1.41 & 1.49 \\
\hline
\end{tabular}




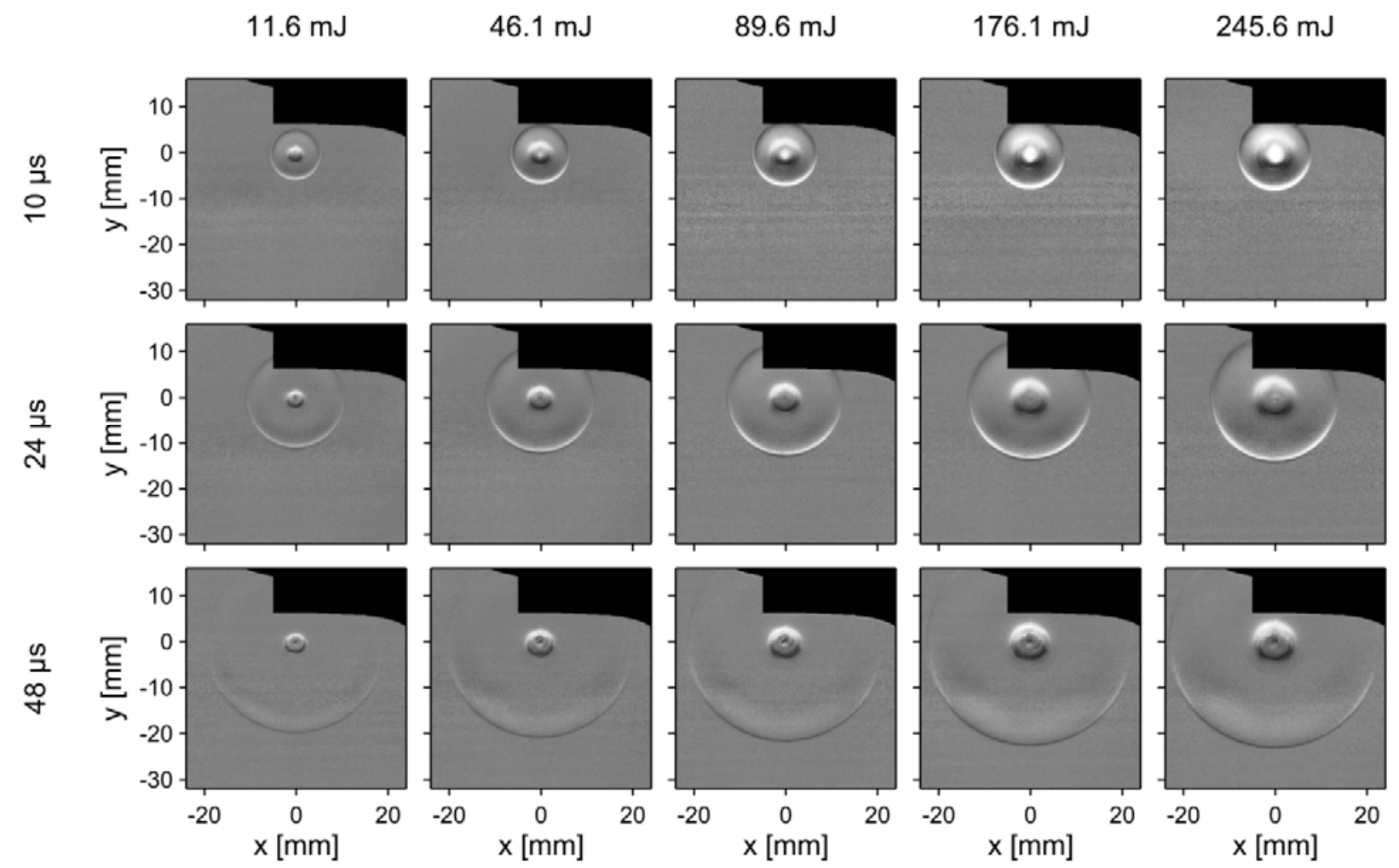

Fig. 4 Representative schlieren images of laser-induced blast waves in air for five different air breakdown energies. The black shape in the upper area is the holder of the doublet lens. The origin is at the focal point of the breakdown.

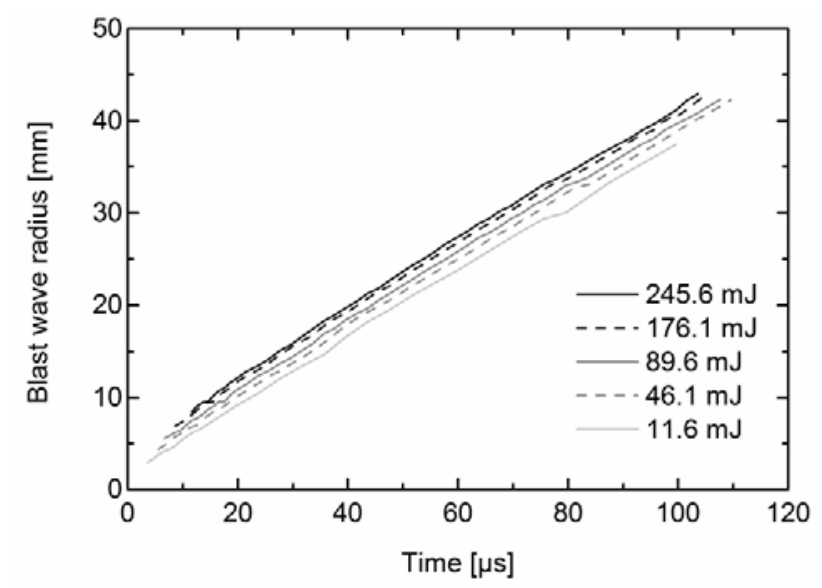

Fig. 5 Tracked blast wave trajectories for five different breakdown energies. 


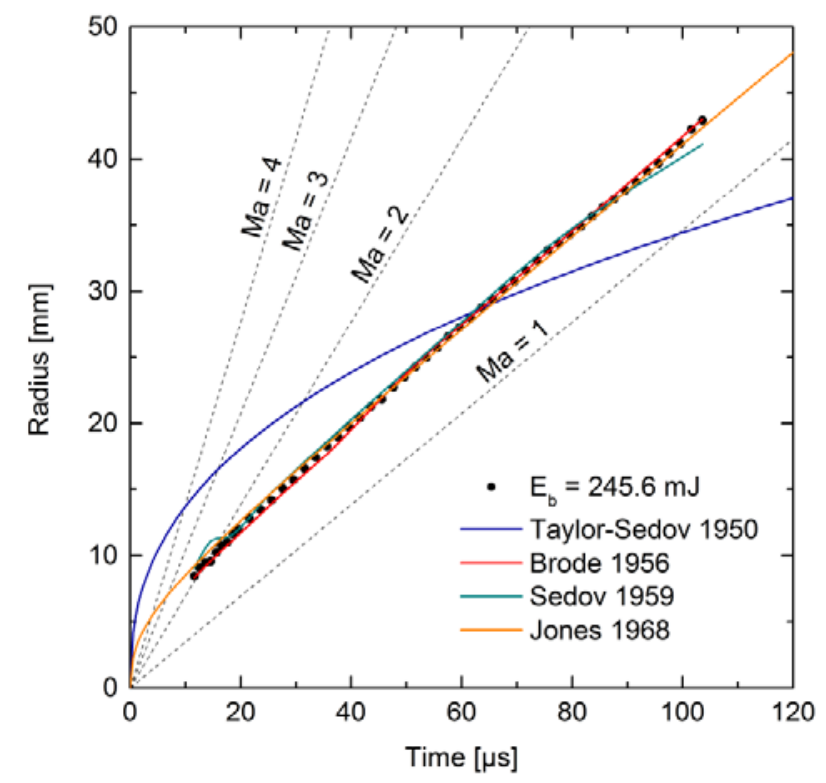

Fig. 6 Comparison of a measured blast wave trajectory with fitted trajectory models.

Tab. 2 Average breakdown and blast wave energies, and sums of least squares of fitted trajectory models. The energy proportions $\left(100 E_{0} / E_{b}\right)$ are given in brackets.

\begin{tabular}{ccccc}
\hline $\begin{array}{c}E_{b} \\
{[\mathrm{~mJ}]}\end{array}$ & $\begin{array}{c}E_{0, \text { Brode }} \\
{[\mathrm{mJ}(\%)]}\end{array}$ & $\begin{array}{c}\Sigma \Delta y_{i, \text { Brode }}{ }^{2} \\
{\left[\mathrm{~mm}^{2}\right]}\end{array}$ & $\begin{array}{c}E_{0, \text { Jones }} \\
{[\mathrm{mJ}(\%)]}\end{array}$ & $\begin{array}{c}\Sigma \Delta y_{i, \text { Jones }}{ }^{2} \\
{\left[\mathrm{~mm}^{2}\right]}\end{array}$ \\
\hline 245.6 & $128.2 \pm 3.2(52.2 \pm 1.3)$ & 4.10 & $125.2 \pm 2.7(51.0 \pm 1.1)$ & 7.73 \\
176.1 & $94.6 \pm 2.5(53.7 \pm 1.4)$ & 3.34 & $95.5 \pm 2.2(54.2 \pm 1.2)$ & 8.95 \\
89.6 & $50.4 \pm 1.4(56.2 \pm 1.6)$ & 1.55 & $52.2 \pm 1.4(58.2 \pm 1.6)$ & 10.56 \\
46.1 & $28.7 \pm 0.9(62.3 \pm 2.0)$ & 2.06 & $29.9 \pm 0.9(64.9 \pm 2.0)$ & 11.28 \\
11.6 & $8.9 \pm 0.4(76.6 \pm 3.4)$ & 1.74 & $9.0 \pm 0.4(77.7 \pm 3.4)$ & 5.13 \\
\hline
\end{tabular}

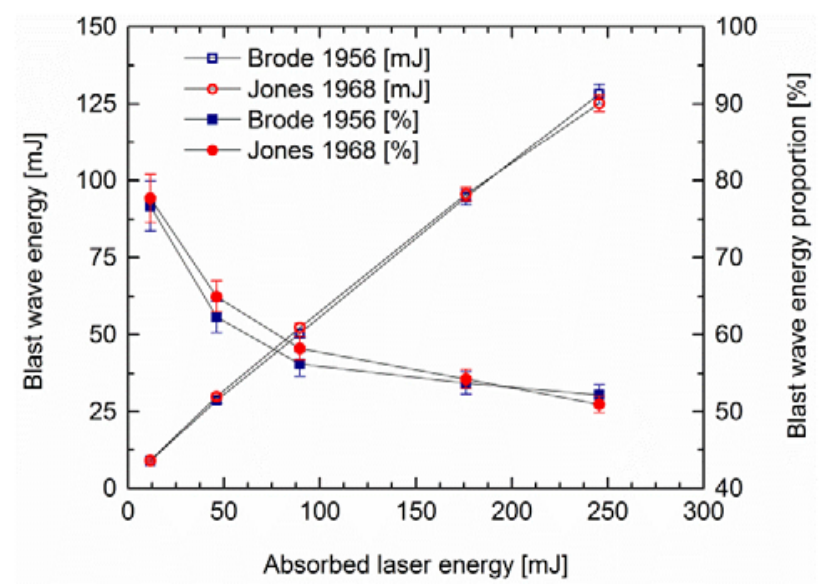

Fig. 7 Average blast wave energies and proportions $\left(100 E_{0} / E_{b}\right)$ for five different breakdown energies. The error bars give the maximum errors within the accuracy of the measurements. 


\subsection{Interaction of blast waves with droplet chains}

In the second part of the experimental investigations, monodisperse ethanol and Jet A-1 kerosene droplets chains were injected into a weak and steady airflow inside the flow channel. The fuel temperature was controlled to be $294 \pm 0.2 \mathrm{~K}$. The air flow was at ambient pressure and temperature, and its mass flow was set to be $215.5 \pm 10.3 \mathrm{~g} / \mathrm{min}$. The purpose of the airflow was to flush fuel vapour out of the measurement area. Although droplet evaporation was negligible at the applied moderate temperatures, the airflow was a precaution to prevent the formation of an ignitable mixture during the experiment. The delay between the laser flash lamp trigger and the Q-switch trigger was kept at $215 \mu \mathrm{s}$, providing the maximum laser pulse energy throughout all measurements. The interaction of the droplet chains with the blast waves was visualized with schlieren and shadowgraph imaging. To obtain a better temporal resolution than the $25 \mathrm{kHz}$ of the high-speed camera, recordings with different delays between the camera start trigger and the laser Q-switch trigger were taken for every investigated experimental configuration. Schlieren and shadowgraph images were recorded for several configurations. Single and five parallel droplet chains of ethanol and a commercial Jet A-1 kerosene (from the international airport of Stuttgart, Germany) were investigated, and the horizontal distance towards the breakdown position was varied to study the effect on the fuel droplets as a function of the blast wave radius. The parameters of the applied droplet chains are given in Table 3 . The required excitation frequency $v_{T S I}$ of the injector and the jet velocity $v_{j e t}$ were calculated with the equations (2) and (3). The droplet diameter $D_{d}$ and the center-to-center distance between two successive droplets $d_{d}$ were calculated with the equations (4) and (5).

Particularly revealing images were taken for five parallel droplet chains, which were positioned between 5 and $9 \mathrm{~mm}$ horizontal distance from the breakdown position. Figure 8 shows three representative schlieren images of kerosene droplet chains 20,40 and $200 \mu$ s after the laser pulse. The horizontal distance between the focal point and the closest droplet chain was $5 \mathrm{~mm}$. At $20 \mu \mathrm{s}$, the plasma radiation from the breakdown is still visible. The laser-induced blast wave has already passed the droplet chains. At $40 \mu \mathrm{s}$, the gas plume has a radius of approximately 4 to $5 \mathrm{~mm}$ and just approaches the first droplet chain. At $200 \mu \mathrm{s}$, the plume continues its expansion and disturbs sections of the first and second droplet chain, marked by the white dashed box. A weak horizontal wave is visible, which is the reflection of the blast wave on the injector plane. The regimes, in which droplets flatten and eventually break up are marked by yellow dashed boxes. They will be in the focus of the forthcoming discussion and analysis in this paper, see section 3.3.

Figure 9 shows shadowgraph images of five parallel kerosene droplet chains recorded 40, 140, 200, and $300 \mu \mathrm{s}$ after the laser pulse. The experimental configuration is exactly the same as in Figure 8 , but the field-of-view is smaller, and density gradients are not visible. The expansion of the gas plume is indicated by a blue circle. All four images show the droplet chains after being struck by the blast 
wave. At $40 \mu \mathrm{s}$, the droplets have deformed to disks. Subsequently they relax and turn into filaments, which are clearly visible at $140 \mu$ s. Filaments at the 5 to $8 \mathrm{~mm}$ horizontal positions show pinchings at $200 \mu \mathrm{s}$, which indicate the beginning breakup. Some droplets at the 5 to $7 \mathrm{~mm}$ positions show two pinchings, while the droplets below and droplets at the $8 \mathrm{~mm}$ position show only one pinching. At the 5 to $7 \mathrm{~mm}$ positions, droplets have disintegrated into two to three secondary droplets at $300 \mu \mathrm{s}$. No breakup occurs at the $8 \mathrm{~mm}$ position. The droplets have recovered from the pinching, and the upper droplets have contracted to disks. Droplets at the $9 \mathrm{~mm}$ position do not show any breakup, but relax from disks into filaments and eventually become spherical again. The different observed deformations and breakups depend on the radial distance from the breakdown origin: Breakup into three secondary droplets occurs within a radius of $7.4 \pm 0.1 \mathrm{~mm}$, and breakup into two secondary droplets occurs within $8.0 \pm 0.1 \mathrm{~mm}$. The radii were determined from images of six individual events, taken 300 and $320 \mu$ s after the laser pulse. They are indicated by the dashed red circles in the right image of Figure 9. Their origin is $1.7 \mathrm{~mm}$ below the breakdown position, which is the distance the droplets have dropped since the blast wave has passed. Breakup does not occur in the upper part of the droplet chains at 5 to $7 \mathrm{~mm}$ distance, whereas it occurs at the lower part. The upper droplets are impacted by the flow field of the expanding gas plume. This apparently overrides their breakup. Instead, it induces distortion and partly coalescence or atomization.

Figure 10 shows similar images, but with ethanol instead of kerosene. The same process is visible, with one exception: Not all droplets at the $8 \mathrm{~mm}$ position recover from the contraction at $200 \mu \mathrm{s}$ but disintegrate into two secondary droplets, which are visible at $300 \mu$ s. Breakup into three secondary droplets occurs within a radius of $7.7 \pm 0.1 \mathrm{~mm}$, and breakup into two secondary droplets within $8.5 \pm 0.1 \mathrm{~mm}$. The greater radii in comparison to kerosene probably resulted from the lower surface tension of ethanol, which is $22.2 \mathrm{mN} / \mathrm{m}$ at $21{ }^{\circ} \mathrm{C}$ [25]. The surface tension of Jet A-1 kerosene is not defined in the fuel specification DEF STAN 91-91 [26], and literature offers different values. For a temperature of $21^{\circ} \mathrm{C}$, the CRC Handbook of Aviation Fuel Properties provides $23.6 \mathrm{mN} / \mathrm{m}$ [27], while Rachner provides $22.8 \mathrm{mN} / \mathrm{m}$ [28]. Therefore, a sample of our Jet A-1 was sent to an external chemical analysis laboratory. The surface tension was measured to be $25.9 \mathrm{mN} / \mathrm{m}$, which was greater than the two literature values. But it still remained within the normal range for kerosenes and similar fuels. It was $16.7 \%$ greater than the surface tension of ethanol, and thus kerosene droplets are more resistant towards aerodynamic breakup.

Figure 11 shows shadowgraph image overlays of single droplet chains $300 \mu$ s after the laser pulse. Images were recorded for ethanol and for kerosene. The different colors emphasize that each image is an overlay of seven individual recordings. The intention of this part of the experiment was to examine if any slipstream effects occur in the configuration with five parallel chains: Droplet chains might diminish the blast-induced flow or the gas plume expansion and consequently influence the breakup of the chains that lie behind. Regarding the gas plume, this was the case: In Figures 9 and 10 the breakup at $7 \mathrm{~mm}$ is not disturbed, contrary to Figure 11. There, the upper part of the chain only shows sporadic breakup, while most of the droplets stay intact and ae partly distorted. Hence, the breakup at the $7 \mathrm{~mm}$ position in the configuration with five chains was not disturbed, because the chains at 5 and 
$6 \mathrm{~mm}$ diminished or even blocked any disruptions by the gas plume. In contrast, the effect of the blast waves on the droplets was the same as in the configuration with one chain. In Figures 10 and 11 breakup into two secondary droplets occurs at the upper part of the ethanol droplet chains at $8 \mathrm{~mm}$. In Figures 9 to 11 breakup into three secondary droplets occurs in the lower part of the kerosene and ethanol droplet chains at $6 \mathrm{~mm}$. In conclusion, the only relevant slipstream effect in the configuration with five chains was the diminishing of the expanding gas plume. Hence, the configuration with five chains was advantageous for the observation of blast wave effects on fuel droplets. The next section provides a closer look at the observed droplet breakup, along with an analysis of the underlying mechanism.

Tab. 3 Controlled $\left(\dot{m}_{f}, v_{T S I}\right)$ and calculated $\left(D_{d}, d_{d}\right)$ parameters of the applied droplet chains.

\begin{tabular}{lcccc}
\hline & $\begin{array}{c}\text { Jet A-1 } \\
\text { One droplet chain }\end{array}$ & $\begin{array}{c}\text { Jet A-1 } \\
\text { Five droplet chains }\end{array}$ & $\begin{array}{c}\text { Ethanol } \\
\text { One droplet chain }\end{array}$ & $\begin{array}{c}\text { Ethanol } \\
\text { Five droplet chains }\end{array}$ \\
\hline$\dot{m}_{f}[\mathrm{~g} / \mathrm{min}]$ & 0.47 & 3.03 & 0.47 & 3.08 \\
$v_{\text {TSI }}[\mathrm{kHz}]$ & 22.36 & 28.96 & 22.68 & 29.37 \\
$D_{d}[\mu \mathrm{m}]$ & 94.5 & 94.3 & 94.2 & 94.5 \\
$d_{d}[\mu \mathrm{m}]$ & 224.9 & 223.9 & 222.7 & 225.1 \\
\hline
\end{tabular}
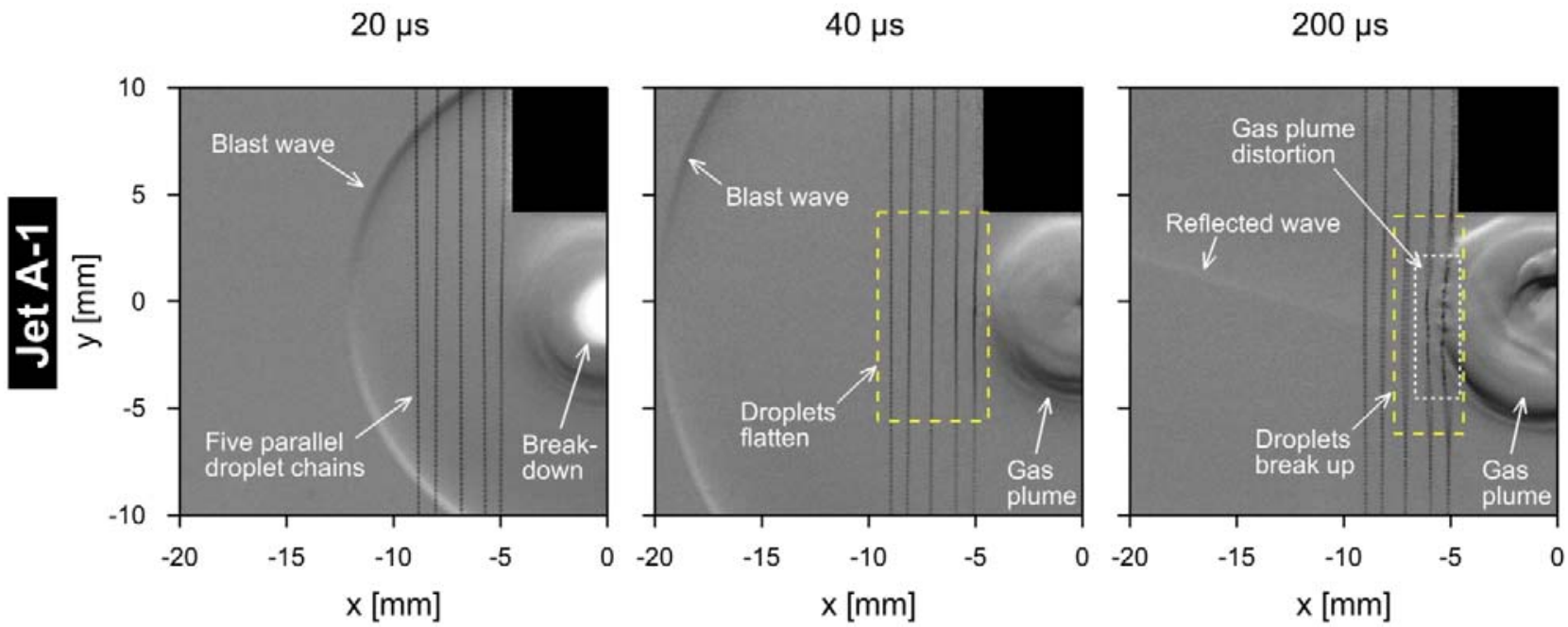

Fig. 8 Schlieren images of five parallel kerosene droplet chains nearby a laser-induced air breakdown. The origin is at the focal point of the breakdown. 


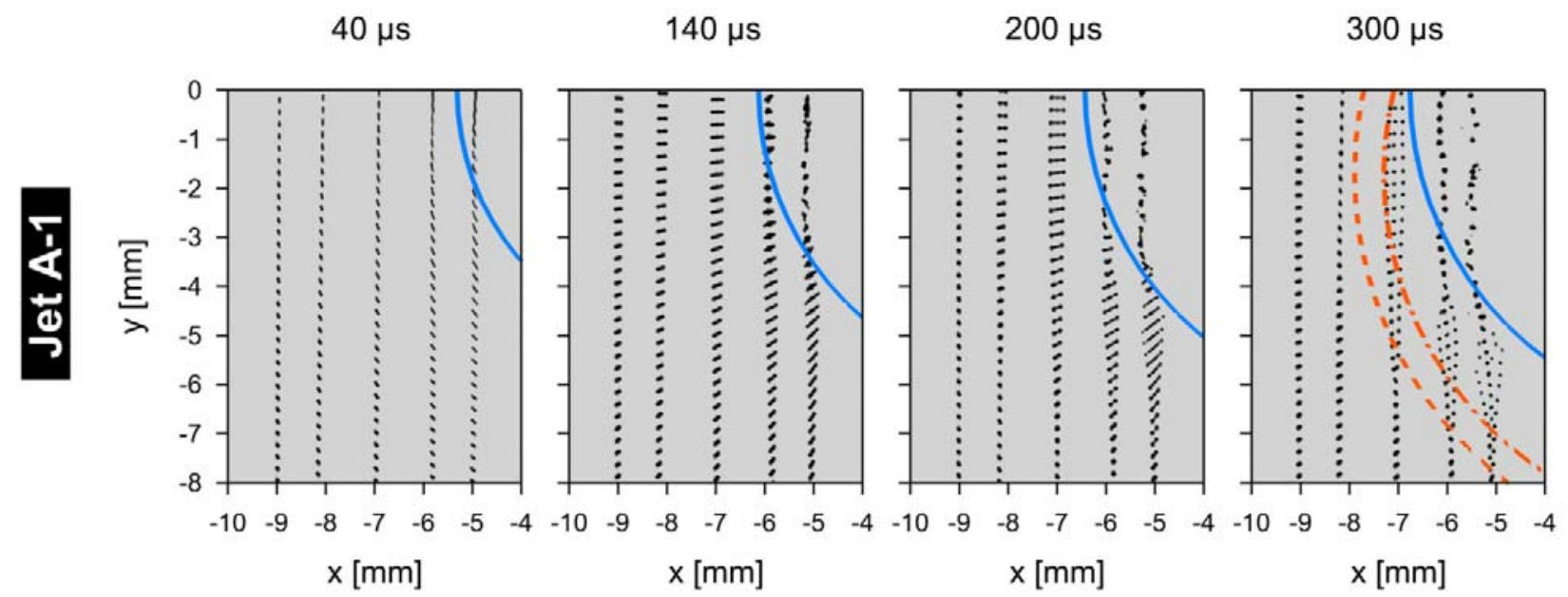

Fig. 9 Shadowgraph image of five parallel kerosene droplet chains. The origin is at the focal point of the breakdown.

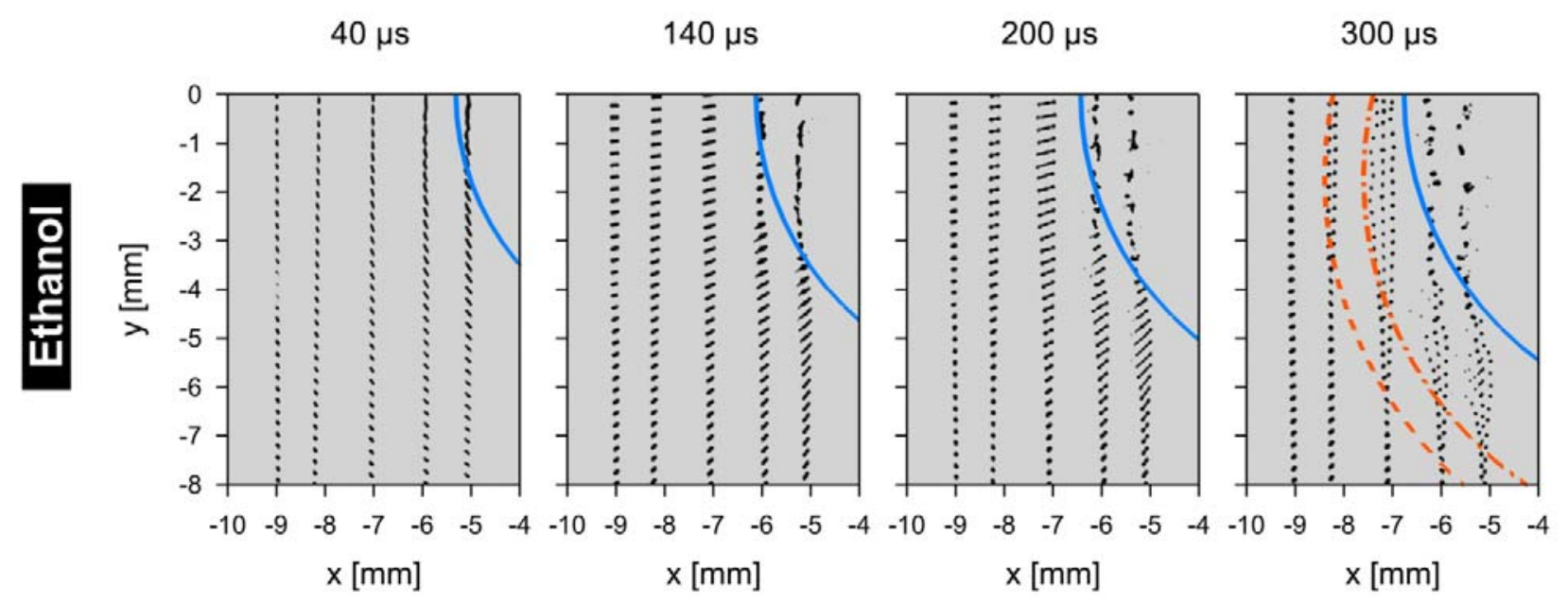

Fig. 10 Shadowgraph image of five parallel ethanol droplet chains. 

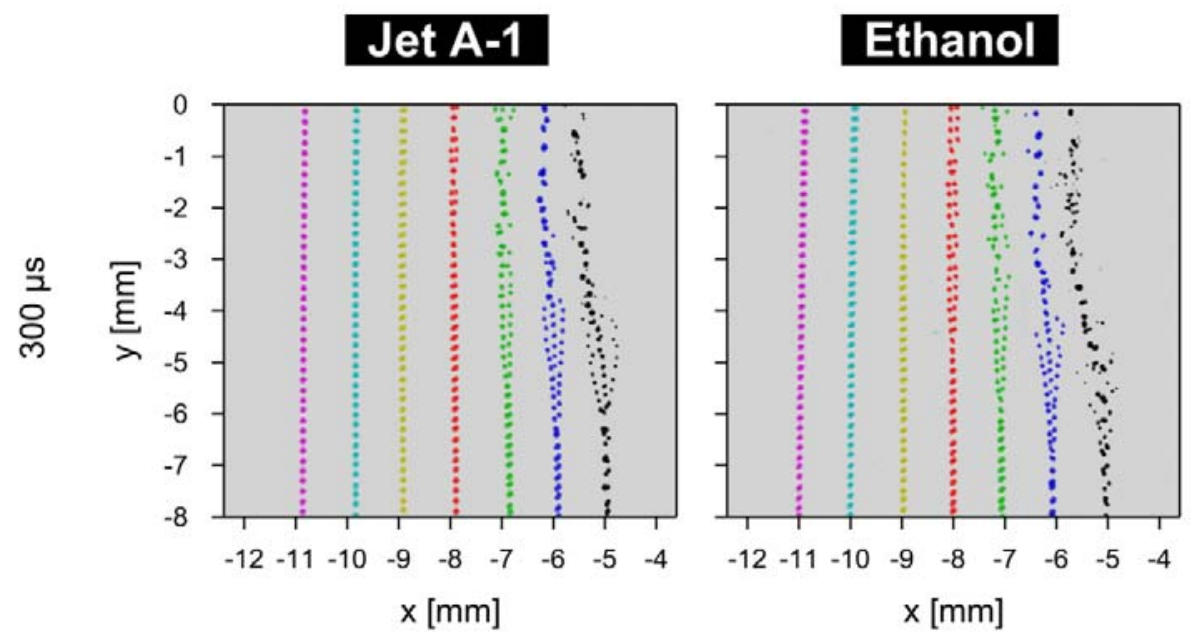

Fig. 11 Overlays of shadowgraph image of single kerosene and ethanol droplet chains. The different colors emphasize that each image is an overlay of seven individual recordings.

\subsection{Analysis}

The shadowgraph images presented in section 3.2 demonstrate that the blast waves induced droplet breakup. Droplets disintegrated into two to three secondary droplets within a radius of 7.9 to $8.4 \mathrm{~mm}$ around the breakdown origin. As the radius increased, the effect diminished. Above $8.4 \mathrm{~mm}$ droplets oscillated but no breakup occured. The Figures 12 and 13 provide enlarged views at 7, 8 and $9 \mathrm{~mm}$ horizontal distance from the breakdown origin for times between 20 and $340 \mu$ s after the laser pulse. Please note: The image sequences show fuel droplets after the blast-induced flow has passed. The analysis in this section shows that in the Eulerian frame of reference the flow decays rapidly. At $20 \mu \mathrm{s}$ after the laser pulse aerodynamic forces, characterized by the Weber number We, are already too weak to have any impact on the droplets. The views in Figures 12 and 13 shift downward by $2 \mathrm{~mm}$ and leftward by 0.1 to $0.2 \mathrm{~mm}$ over time. The shift compensates gravitation-induced descent of the droplets and their leftward motion initiated by the aerodynamic drag of the blast-induced flow. Hence, the enlarged views only show droplets which were approximately at the height of the breakdown when they were hit by the blast wave. The droplet deformation and breakup occur as described in section 3.2. Droplet breakup into three secondary droplets occurs at the $7 \mathrm{~mm}$ position. At the $8 \mathrm{~mm}$ position ethanol droplets break up into two secondary droplets, while kerosene droplets remain intact but oscillate linearly. At the $9 \mathrm{~mm}$ position, ethanol and kerosene droplets oscillate linearly but remain intact. Linear oscillation and droplet breakup take place along a nearly horizontal axis. This results from the local expansion direction of the blast wave, which hit the droplets from the left and proceeded to the right.

Several recordings with different delays between the Q-switch trigger and the camera start were required to overcome the limited camera repetition rate. The sequences shown in Fig. 12 and 13 were put together from three individual recordings of the experiment. Although the reproducibility was 
very good, small differences could occur. Please see for instance the droplet filaments at the $7 \mathrm{~mm}$ position at $200 \mu \mathrm{s}$ in Fig. 13. Their tilt angles differ from their neighbors at 180 and $220 \mu$ s (both taken in the same recording) by 4.5 degrees. This does not result from a rotational oscillation but from small, unidentified differences between the two recordings of the same experiment. However, no differences between the transient morphologies of the deformations and breakup processes were observed, and they are well represented by the sequences.

The enlarged views assist the analysis of the underlying mechanism. On the post-shock side, blast waves feature strong, but quickly decreasing blast-induced flows. At present, there is no analytical method available to characterize droplet deformation and breakup in such flows. However, the application of the methods developed for steady flows can provide insightful information. Droplet breakup in steady flow fields is commonly characterized by the Weber number $W e$ and the Ohnesorge number $\mathrm{Oh}$. The impact of the latter is negligible if $O h<0.1$ [7], which is true for the herein presented experiments. The Weber number is defined as

$$
W e=\frac{u_{g}^{2} \rho_{g} D_{d}}{\sigma_{d}}
$$

Here, $u_{g}$ is the velocity of the gas flow field, $\rho_{g}$ is the density of the gas, and $\sigma_{d}$ is the droplet surface tension. It is very difficult to measure blast-induced flow velocities, but they can be numerically determined when the blast wave energy is known. The energy determination is reported in section 3.1. It shows that the blast waves absorb approximately $52 \%$ of the breakdown energy at the energy regime applied in the experiments presented in section 3.2. One of the successfully applied blast wave models in section 3.1 is the non-dimensional numerical simulation by Brode [19]. Another simulation by Brode provides non-dimensional densities and velocities of blast-induced flows [29]. The diagrams from his paper were digitized, and the transient flow velocities and Weber numbers at 5 , $6,7,8$ and $9 \mathrm{~mm}$ radial distance from the breakdown origin were interpolated. A blast wave energy of $128.2 \mathrm{~mJ}$ was applied, which is the energy determined with Brode's model in section 3.1. Figure 14 gives the flow velocities for the five investigated radii, and Figure 15 gives the corresponding droplet Weber numbers. The latter were calculated for ethanol and kerosene droplet diameters of 94.5 and $94.3 \mu \mathrm{m}$, respectively, according to Table 3. Primarily due to the different surface tensions, kerosene Weber numbers are smaller by approximately $17 \%$ compared to ethanol Weber numbers. The diagrams also show that the post-shock flow velocities decrease towards zero within approximately $10 \mu \mathrm{s}$ after the shock front has passed. The subsequent negative flow velocities indicate the rarefaction waves, which are considerably weaker and last for approximately $25 \mu \mathrm{s}$.

Pilch and Erdman [5] reviewed experimental studies of droplet breakup in steady flow fields. They identified five different breakup modes, which are determined by the magnitude of the Weber number. According to Guildenbrecher et al. [7], the extremes are the vibrational breakup for $W e<11$, where droplets disintegrate due to resonant excitation, and the catastrophic breakup for $W e>350$, where 
droplets atomize into very small secondary droplets. Several breakup mechanisms exist in between, such as the bag breakup and the sheet thinning breakup. Experimental and numerical studies consistently show that for We $>11$ droplet breakup in steady flows typically begins with a deformation into a flat shape, such as a disk, lens or bowl, see for instance the publications $[10,12,30,31]$. Extensive atomization of the surfaces of still spherical droplets occurs only for very high Weber numbers at the order of 1000. A comparison of the known breakup mechanisms with Fig. 12 and 13 provides an understanding of the observed process. Table 4 provides supporting information. It gives the blast wave arrival time after the laser pulse and the Weber numbers $0.1 \mu \mathrm{s}$ later. At all three positions, 7 to $9 \mathrm{~mm}$, the Weber numbers are initially well above 11 and therefore, for a short time, aerodynamic forces are sufficient to deform the droplets into flat shapes. The result of this aerodynamic flattening can be seen clearly at $20 \mu \mathrm{s}$ in Fig. 12 and 13. The flattening has proceeded at $40 \mu \mathrm{s}$. At this time, the flow field has already turned into a weak rarefaction wave, according to Figure 14. In the associated Fig. 15 the Weber numbers are well below 1, aerodynamic forces are too weak to have any impact on the droplets. Thus, the proceeding flattening of the droplets results from previously transmitted kinetic energy from the blast-induced flow, which now turns into strain energy. The following sequences in Fig. 12 and 13 are different for the two fuels and the individual horizontal positions. At the $9 \mathrm{~mm}$ position both, kerosene and ethanol droplets do not disintegrate, but oscillate.

The angular fundamental frequencies of a spherical droplet surrounded by a gaseous medium are [32]

$$
\omega_{0, n}=\left[\frac{(n-1) n(n+1)(n+2) \sigma_{f}}{\left[n \rho_{g}+(n+1) \rho_{f}\right] R_{d}^{3}}\right]^{\frac{1}{2}},
$$

where $R_{d}$ is the droplet radius and $n \geq 2$ is the fundamental mode. For $n=2$ droplets feature a linear, axisymmetric oscillation at the ordinary fundamental frequency

$$
v_{0}=\frac{\omega_{0,2}}{2 \pi}=\left[\frac{6 \sigma_{f}}{\left(2 \rho_{g}+3 \rho_{f}\right) \pi^{2} R_{d}{ }^{3}}\right]^{\frac{1}{2}} .
$$

For the fuel densities $\rho_{f, \text { kerosene }}=793.4 \mathrm{~kg} / \mathrm{m}^{3}$ and $\rho_{f \text {, thanol }}=789,8 \mathrm{~kg} / \mathrm{m}^{3}$ and the air density of $\rho_{g}=1.21 \mathrm{~kg} / \mathrm{m}^{3}$ the ordinary frequencies calculate to be $v_{0, \text { kerosene }}=7940.1 \mathrm{~Hz}$ and $v_{0, \text { ethanol }}=7367.8 \mathrm{~Hz}$. The frequencies correspond to periods of $T_{0, \text { kerosene }}=125.9 \mu \mathrm{s}$ and $T_{0 \text {,thanol }}=135.7 \mu \mathrm{s}$. At the $9 \mathrm{~mm}$ position in Fig. 12, kerosene droplets are flat at $40 \mu \mathrm{s}$, but subsequently expand into spheres at $80 \mu$ s. The expansion proceeds and is followed by a contraction. 
Droplets again feature a spherical shape at $200 \mu \mathrm{s}$, followed by another flattening. Finally, at $340 \mu \mathrm{s}$ they are almost spherical again. It seems that a second linear expansion just begins, which means that the spherical shape is reached shortly before $340 \mu \mathrm{s}$. Shadowgraph images are available every $20 \mu \mathrm{s}$, therefore periods are determined with an accuracy of $\pm 10 \mu \mathrm{s}$. A spherical droplet shape at 80, 200 and $<340 \mu$ s indicates a periodic length of approximately $120 \pm 10 \mu$ s. The same analysis for ethanol droplets indicates a periodic length of $140 \pm 10 \mu \mathrm{s}$ with a spherical droplet shape at 80 and $220 \mu \mathrm{s}$. The agreement with the calculated fundamental frequencies is very good. It indicates the existence of a transient oscillation, which results from a preceding external impulse excitation.

The image sequence from 180 to $220 \mu \mathrm{s}$ at the 7 and $8 \mathrm{~mm}$ position in Figures 12 and 13 indicates that capillary forces play a crucial role for the droplet breakup. After having expanded linearly, droplets feature filament-like shapes. The filaments have diameters of only several tens of microns and therefore are prone to capillary pinching. At the $8 \mathrm{~mm}$ position, single pinchings occur at the centers of the filaments. Kerosene droplets recover from the pinchings and continue to oscillate without disintegration. Due to their lower surface tension, ethanol droplets at the $8 \mathrm{~mm}$ position disintegrate and form two secondary droplets with a uniform diameter, see Fig. 13 at $340 \mu \mathrm{s}$. At the $7 \mathrm{~mm}$ position, both kerosene and ethanol droplet filaments feature two pinchings, resulting in the pinch-offs of two secondary droplets after $220 \mu \mathrm{s}$. A comparison of Fig. 12 and 13 at $200 \mu$ s shows that the ethanol filaments stretch more than the kerosene filaments. Again, this is a result of the lower surface tension of ethanol. Interestingly, the three secondary droplets of both, kerosene and ethanol appear to be of nearly uniform diameters. Highly resolved images of the disintegrating droplets are provided in our previous publication [3]. For an in-depth description of droplet breakup by capillary pinching, the reader is referred to the investigation by Tjahjadi et al. [33] and the review paper by Stone [34].

In conclusion, the herein observed droplet breakup proceeds as follows: When droplets are exposed to spherical blast waves from laser-induced breakdowns, they experience strong aerodynamic forces which initiate flattening. The flow velocities decrease quickly. According to Fig. 15, Weber numbers decrease below 1 within $10 \mu$ s after the arrival of the blast wave. Therefore, breakups do not result from instantaneous aerodynamic forces, which is the case in steady flow fields. When the Weber numbers have decreased, droplets continue to flatten for several microseconds due to kinetic energy previously transmitted by the flow fields. As soon as the kinetic energy has completely turned into strain energy, the flattening terminates. Subsequently, the strain energy is released, the droplets relax and stretch into filaments. The stretching rate depends on the level of stored strain energy, and therefore is a function of the integral strength of the flow field, which becomes weaker with increasing distance from the breakdown origin. Consequently, at closer distances filaments are longer and thinner than at further distances. Thinner filaments are more prone to capillary pinching. Therefore, filaments within a certain distance from the breakdown origin disintegrate by pinching in the center of the filament or by pinch-offs of two secondary droplets at the filament ends. Filaments at greater radial distances, which are loaded with less strain energy, may also feature pinching, but subsequently contract and oscillate for several periods. In principle, this process is similar to the vibrational breakup 
in steady flows at $W e<11$ by the fact that droplets stretch into filaments and disintegrate by capillary pinching. The difference is that stretching in steady flows results from the buildup of a linear oscillation. Here, the critical stretching is reached after a single, impulse-like excitation.

Tab. 4 Arrival time of the blast wave after the laser pulse and post-shock flow Weber numbers $0.1 \mu \mathrm{s}$ after the arrival of the blast wave.

\begin{tabular}{lccc}
\hline & $7 \mathrm{~mm}$ & $8 \mathrm{~mm}$ & $9 \mathrm{~mm}$ \\
\hline Blast wave arrival time $t_{1}[\mu \mathrm{s}]$ & 8.5 & 10.7 & 13.1 \\
$W e_{\text {kerosene }}\left(t_{1}+0.1 \mu \mathrm{s}\right)$ & 159.4 & 117.6 & 66.7 \\
$W e_{\text {ethanol }}\left(t_{1}+0.1 \mu \mathrm{s}\right)$ & 186.1 & 137.4 & 78.0 \\
\hline
\end{tabular}

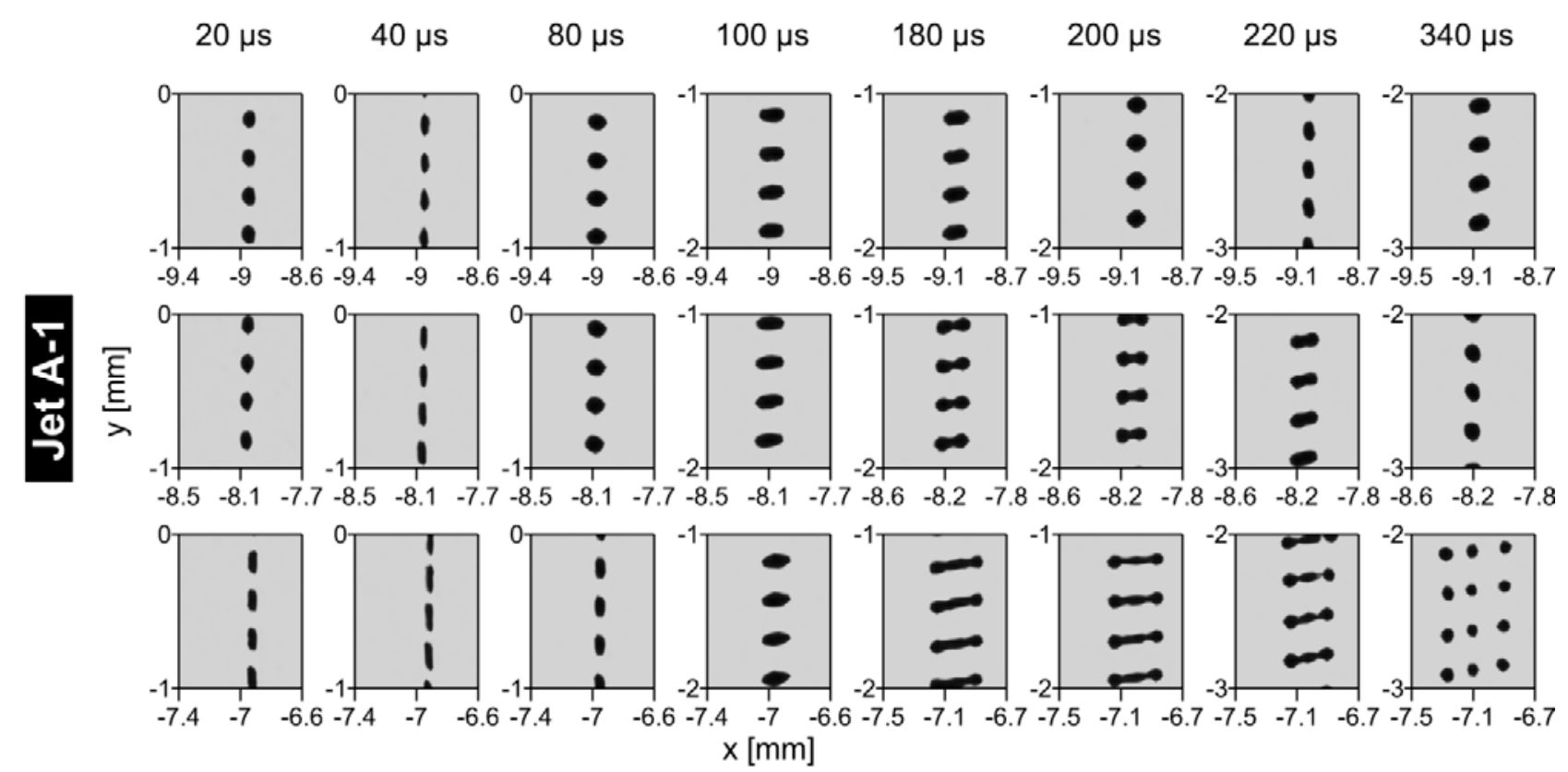

Fig. 12 Enlargement of kerosene droplets in the shadowgraph images at 7,8 and $9 \mathrm{~mm}$ horizontal position. The blast-induced flow has passed from right to left before the images were taken. The origin is at the focal point of the breakdown. The sequences are put together from three individual recordings of the same experiment. 


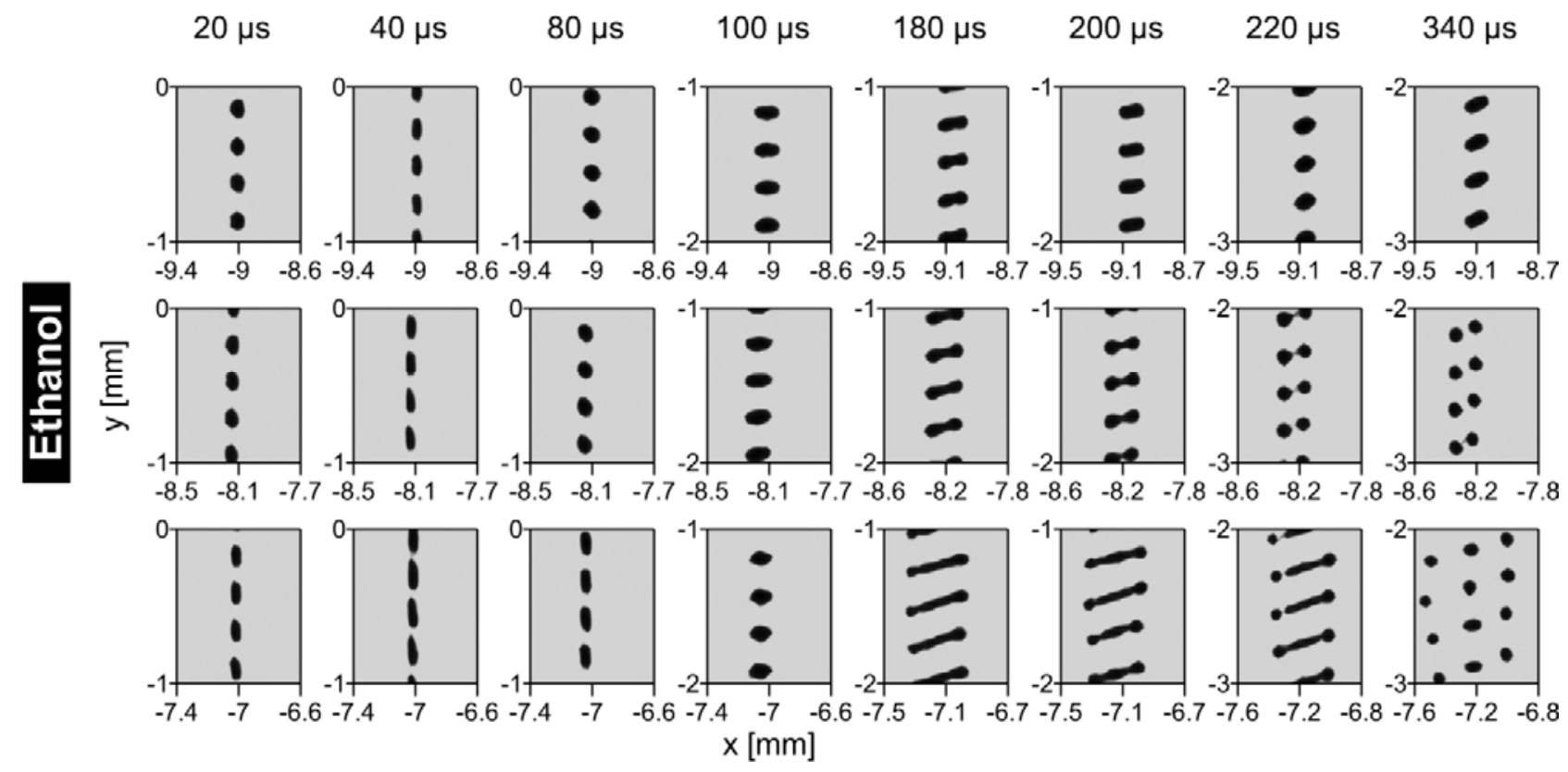

Fig. 13 Enlargement of ethanol droplets in the shadowgraph images at 7,8 and $9 \mathrm{~mm}$ horizontal position. The sequences are put together from three individual recordings of the same experiment.

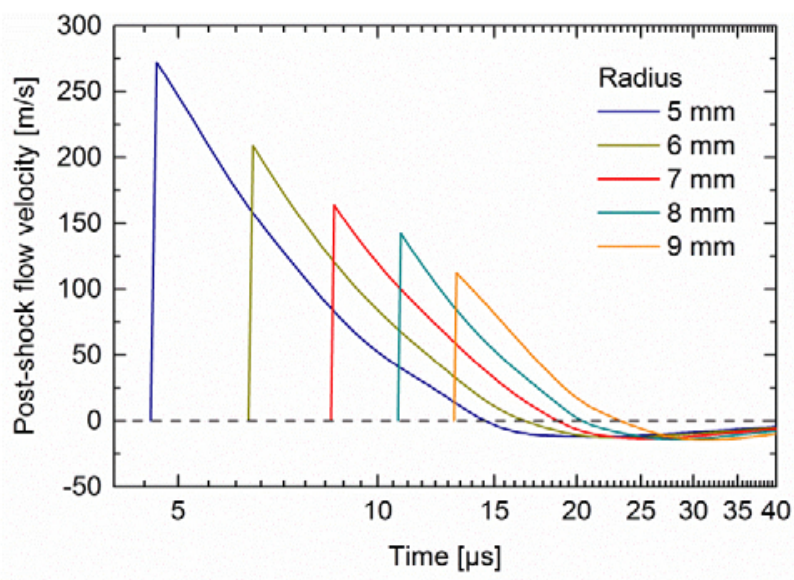

Fig. 14 Calculated post-shock flow velocities for different radii around the breakdown origin. Positive velocities stem from expansion behind the shock front, negative velocities stem from the rarefaction wave. 


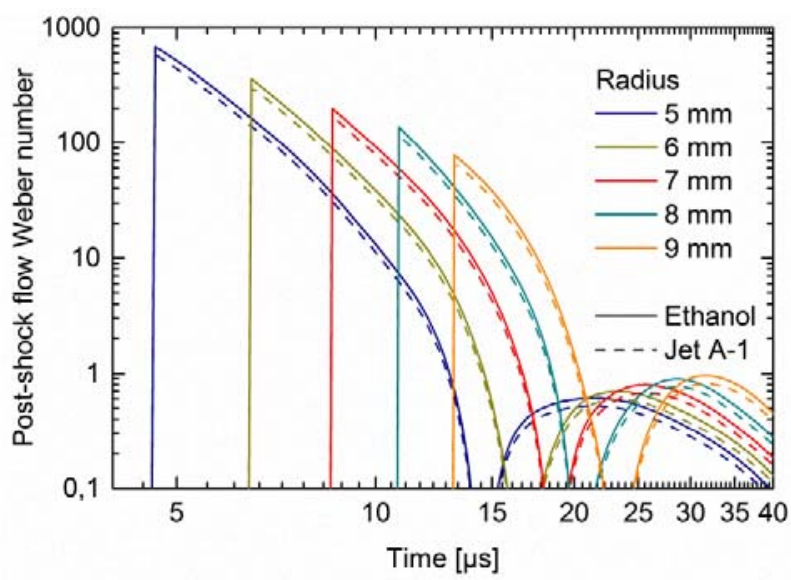

Fig. 15 Calculated post-shock droplet Weber numbers for different radii around the breakdown origin.

\section{Summary and conclusion}

Blast waves were generated by laser-induced air breakdowns. They were visualized with schlieren imaging, and their expansion trajectories were tracked. Theoretical blast wave models by Taylor, Sedov, Brode and Jones were fitted to the trajectory of a blast wave created by a breakdown with an energy of $245.6 \mathrm{~mJ}$. The initial blast wave energy was determined, and the qualities of the fits were evaluated by the sums of the least $\Delta y$-squares. The models by Brode and Jones gave very good fits and reasonable results. They were subsequently validated over a large range of energies by their comparative application to five individual blast wave trajectories from breakdowns with energies between 11.6 and $245.6 \mathrm{~mJ}$. Both models gave similar results: blast waves consume significant portions of the breakdown energies, which increase with decreasing breakdown energy. Approximately $52 \%$ was transferred into the blast waves at a breakdown energy of $245.6 \mathrm{~mJ}$, while $77 \%$ was transferred at $11.6 \mathrm{~mJ}$. The effect of the blast waves on ethanol and kerosene fuel droplets was visualized with shadowgraph imaging. Droplets with a diameter of approximately $94.5 \mu \mathrm{m}$ disintegrated into two to three secondary droplets within a radius of several millimeters around the breakdown position. Ethanol droplets disintegrated into three secondary droplets within $7.7 \mathrm{~mm}$ and into two secondary droplets between 7.7 and $8.5 \mathrm{~mm}$. Due to the higher surface tension, the radii were smaller for kerosene. Disintegration into three secondary droplets occured within $7.4 \mathrm{~mm}$ and into two secondary droplets between 7.4 and $8.0 \mathrm{~mm}$. The given radii are averages from six individual images, the variation is $\pm 0.1 \mathrm{~mm}$. The calculation of the transient post-shock flow velocities and Weber numbers showed that initial Weber numbers behind the shock front were of the order of 100, but decreased below 1 within $10 \mu$ s. Therefore the aerodynamic forces behind the shock front were strong enough to deform the droplets into disks and to initiate, but not complete, breakup. We concluded that the blast-induced flow was only responsible for the droplet deformation into disks, but the subsequent breakup resulted from the release of strain energy. Disk-like droplets relaxed and stretched into filaments. Depending on the initial Weber numbers, e.g. the loaded strain energy, droplets either oscillated over several periods or disintegrated within $220 \mu \mathrm{s}$. Oscillating droplets at $9 \mathrm{~mm}$ distance 
from the breakdown origin featured a period of $120 \pm 10 \mu \mathrm{s}$ (kerosene) and $140 \pm 10 \mu \mathrm{s}$ (ethanol). Theoretical fundamental periods were calculated to be $125.9 \mu \mathrm{s}$ (kerosene) and $135.7 \mu$ s (ethanol), which was in very good agreement with the measured periods and indicated the presence of a transient droplet oscillation after a single, impulse-like excitation. Disintegration into two and three secondary droplets of nearly uniform size resulted from pinching of the filaments due to capillary forces. The experiments helped to understand the role of blast waves in fuel spray ignition. Blast waves can induce breakup of the fuel droplets into smaller secondary droplets within a radius of several millimeters around the spark location. Those secondary droplets can evaporate faster than the initial ones and therefore contribute to the generation of a combustible gas mixture, in which a spray flame kernel can grow and stabilize. To consider this effect in future numerical spray ignition simulations, further investigations of the herein presented breakup mechanism are recommended with both, experimental and numerical methods. Our herein presented experiments were performed with great thoroughness, and results may be used as validation data for numerical simulations.

\section{References}

[1]

Ronney, P.D.: Laser versus Conventional Ignition of Flames. Opt. Eng. 33, 510-521 (1994)

[2]

Gebel, G.C., Mosbach, T., Meier, W., Aigner, M.: Optical and spectroscopic diagnostics of laserinduced air breakdown and kerosene spray ignition, Combust. Flame (2014).

doi:10.1016/j.combustflame.2014.11.024

[3]

Gebel, G.C., Le Brun, S., Mosbach, T., Meier, W., Aigner, M.: An Experimental Investigation of Kerosene Droplet Breakup by Laser-Induced Blast Waves. J. Eng. Gas Turb. Power 135, 021505 (2013)

[4]

Joarder, R., Gebel, G.C., Mosbach, T.: Two-dimensional Numerical Simulation of a Decaying Laser Spark in Air with Radiation Loss. Int. J. Heat. Mass Transf. 63, 284-300 (2013)

[5]

Pilch, M., Erdman, C.A.: Use of Breakup Time Data and Velocity History Data to Predict the Maximum Size of Stable Fragments for Acceleration-Induced Breakup of a Liquid Drop. Int. J. Multiphase Flow 13, 741-757 (1987) 
[6]

Gelfand, B.E.: Droplet Breakup Phenomena in Flows with Velocity Lag. Prog. Energy Combust. Sci. 22, 201-265 (1996)

[7]

Guildenbrecher, D.R., López-Rivera, C., Sojka, P.E.: Secondary atomization. Exp. Fluids 46, 371-402 (2009)

[8]

Engel, O.G.: Fragmentation of Waterdrops in the Zone Behind an Air Shock. J. Research Nat. Bureau Stand. 60, 245-280 (1958)

[9]

Ranger, A.A., Nicholls, J.A.: Aerodynamic Shattering of Liquid Drops. In: Proc. $6^{\text {th }}$ AIAA Aerospace Science Meeting, 22-24 January, New York, NY, AIAA 68-83 (1968)

[10]

Hsiang, L.-P., Faeth, G.M.: Drop Deformation and Breakup due to Shock Wave and Steady Disturbances. Int. J. Multiphase Flow 21, 545-560 (1995)

[11]

Widdecke, N., Klenk, W., Frohn, A.: Impact of Strong Shock Waves on Monodisperse Isopropanol Droplet Streams. In: Brun, R., Dumitrescu, L.Z. (eds.) Shock Waves @ Marseille III, pp 89-94. Springer, Berlin (1995)

Theofanous, T.G., Li, G.J., Dinh, T.N.: Aerobreakup in Rarefied Supersonic Gas Flows. J. Fluids Eng. 126, 516-527 (2004)

Strutt, J.W., $3^{\text {rd }}$ Baron Rayleigh: On the Instability of Jets. Proc. London Math. Soc. 10, 4-13 (1878)

Chen, Y.-L., Lewis, J.W.L., Parigger, C.: Spatial and temporal profiles of pulsed laser-induced air plasma emissions. J. Quant. Spectrosc. Radiat. Transf., 67, 91-103 (2000)

[15]

Phuoc, T.X.: Laser-induced spark ignition fundamental and applications. Opt. Lasers Eng. 44, 351397 (2006) 
Taylor, G.: The Formation of a Blast Wave by a Very Intense Explosion. I. Theoretical Discussion. Proc. R. Soc. Lond. A 201, 159-174 (1950)

Taylor, G.: The Formation of a Blast Wave by a Very Intense Explosion. II. The Atomic Explosion of 1945. Proc. R. Soc. Lond. A 201, 175-186 (1950)

[18]

Sedov, L.I.: Similarity and Dimensional Methods in Mechanics. Academic Press, New York (1959)

[19]

Brode, H.L.: Point Source Explosion in Air. Report RM-1824-AEC, The RAND Corporation (1956)

[20]

Jones, D.L.: Intermediate Strength Blast Waves. Phys. Fluids 11, 1664-1667 (1968)

[21]

Jones, D.L.: Erratum: Strong Blast Waves in Spherical, Cylindrical, and Plane Shocks. Phys. Fluids 5, $637(1962)$

[22]

Brieschenk, S., Hruschka, R., O'Byrne, S., Kleine, H.: High-speed time-resolved visualization of laser-induced plasma explosions. $28^{\text {th }}$ International Congress on High-Speed Imaging and Photonics, 9-14 November 2008, Canberra, Australia, SPIE Vol. 7126 (2009)

[23]

Dewey, J.M.: Expanding spherical shocks (blast waves). In: Ben-Dor, G., Igra, O., Elperin, T. (eds.) Handbook of Shock Waves, Vol 2 - Shock Wave Interactions and Propagation, pp. 441-481. Academic Press, New York (2001)

Phuoc T.X., White, F.P.: An Optical and Spectroscopic Study of Laser-Induced Sparks to Determine Available Ignition Energy. Proc. Combust. Inst. 29, 1621-1628 (2002)

[25]

Vázquez, G., Alvarez, E., Navaza, J.M.: Surface Tension of Alcohol + Water from 20 to $50{ }^{\circ} \mathrm{C}$. J. Chem. Eng. Data 40, 611-614 (1995) 
[26]

Defence Standard 91-91, Issue 7. UK Ministry of Defence (2011)

[27]

Handbook of Aviation Fuel Properties, Third Edition. Report 635, Coordinating Research Council (2004)

[28]

Rachner, M.: The Properties of Kerosine Jet A-1. Report 98-01, German Aerospace Center (1998)

[29]

Brode, H.L.: Numerical Solutions of Spherical Blast Waves. J. Appl. Phys. 26, 766-775 (1955)

[30]

Hinze, J.O.: Fundamentals of the Hydrodynamic Mechanism of Splitting in Dispersion Processes. Am. Inst. Chem. Eng. J. 1, 289-295 (1955)

[31]

Khare, P., Ma, D., Chen, X., Yang, V.: Phenomenology of Secondary Breakup of Newtonian Liquid Droplets. In: Proc. 50 ${ }^{\text {th }}$ AIAA Aerospace Science Meeting, 9-12 January, Nashville/Tennessee USA, AIAA 2012-0171 (2012)

[32]

Ashgriz, N., Movassat, M.: Oscillation of Droplets and Bubbles. In Ashgriz, N. (ed.) Handbook of Atomization and Sprays, pp. 125-144. Springer, Berlin (2011)

[33]

Tjahjadi, M., Stone, H.A., Ottino, J.M.: Satellite and Subsatellite Formation in Capillary Breakup. J. Fluid Mech. 243, 297-317 (1992)

[34]

Stone, H.A.: Dynamics of Drop Deformation and Breakup in Viscous Fluids. Anu. Rev. Fluid Mech. 26, 65-102 (1994) 